\title{
Multishell model for Majumdar-Papapetrou spacetimes
}

\author{
Metin Gürses* \\ Mathematics Department, Bilkent University, 06800 Ankara-Turkey
}

Burak Himmetoḡlu ${ }^{\dagger}$

Physics Department, Bilkent University, 06800 Ankara-Turkey

(Received 4 May 2005; published 29 July 2005; publisher error corrected 2 August 2005)

\begin{abstract}
Exact solutions to static and nonstatic Einstein-Maxwell equations in the presence of extremely charged dust embedded on thin shells are constructed. Singularities of multi-black-hole Majumdar- Papapetrou and Kastor-Traschen solutions are removed by placing the matter on thin shells. Double spherical thin shell solution is given as an illustration and the matter densities on the shells are derived.
\end{abstract}

DOI: 10.1103/PhysRevD.72.024032

PACS numbers: 04.40.Nr, 04.20.Jb

\section{INTRODUCTION}

It was shown that the Majumdar-Papapetrou (MP) solution [1,2] describes a spacetime possessing $N$ extremely charged black holes [3]. Later, extremely charged static dust sources for MP spacetimes were considered [4,5] and it was shown that solutions to Einstein-Maxwell equations with extremely charged dust restricted to thin shells exist. Moreover, solutions for various geometries including planar, spherical and cylindrical shells were obtained [5]. It was also shown that spherical shells can be used as sources for the Extreme Reissner-Nordström (ERN) spacetimes [5]. The cosmological black-hole solutions which are time-dependent generalizations of the MP solution were also studied [6,7] and generalizations with discussions on nonradiative character of these spacetimes were discussed in [8].

In linear theories mass density of point particle distribution can be expressed as $\rho(\mathbf{r})=\sum_{i} m_{i} \delta\left(\mathbf{r}-\mathbf{a}_{\mathbf{i}}\right)$ where $m_{i}$ 's are the masses of each particle, $\delta(\mathbf{r})$ is the Dirac delta function and $a_{i}$ 's are the locations of the point particles. This expression is in consistence with the Poisson's equation,

$$
\nabla^{2} V=-4 \pi \rho \quad \text { with } \quad V=\sum_{i=1}^{N} \frac{m_{i}}{\left|\mathbf{r}-\mathbf{a}_{\mathbf{i}}\right|}
$$

Such a point particle representation of the mass density is not valid in nonlinear theories. For instance, in a nonlinear theory like $\nabla^{2} V+4 \pi \rho V^{3}=0$, there exists no solution $V$ for $\rho=\sum_{i} m_{i} \delta\left(\mathbf{r}-\mathbf{a}_{\mathbf{i}}\right)$. We overcome the above problem by replacing massive particles by spherical thin shells with centers at $\mathbf{r}=\mathbf{a}_{\mathbf{i}}$ and radii $r_{i}$. Then the mass density $\rho$ can now be represented as $\rho(\mathbf{r})=\sum_{i=1}^{N} \rho_{0 i} \delta\left(F_{i}\right)$ where $F_{i}(\mathbf{r})=0(i=1,2, \ldots, N)$ represents the positions of the shells and $\rho_{0 i}$ are the mass density of each thin shell. The space is divided into $N+1$ spaces. In our proposed

\footnotetext{
*Electronic address: gurses@fen.bilkent.edu.tr
}

†Electronic address: himmet@ug.bilkent.edu.tr method we shall solve such potential problems where the solutions are continuous everywhere.

In this work, we will first review the solutions to Einstein-Maxwell equations in the presence of extremely charged static dusts restricted to thin shells that are given in [5]. Then we will generalize the methods in [5] to find solutions possessing extremely charged dusts restricted to multiple shells and obtain singularity free versions of MPsolutions given in [3]. We will also work out the double shell problem explicitly and show that the matter distributions on the shells are not uniform and that interior of the shells are not flat unlike the single shells in [5]. Finally we will work on cosmological MP solutions discussed in [6$8]$ and develop similar multishell models.

\section{SHELL MODELS FOR THE MAJUMDAR- PAPAPETRAU SPACETIMES}

Let $\mathcal{M}$ be a four dimensional spacetime with the metric,

$$
d s^{2}=-\lambda^{-2} d t^{2}+\lambda^{2} h_{i j} d x^{i} d x^{j},
$$

where $h_{i j}$ is an Euclidean 3-metric and $\lambda$ is a function of spatial coordinates $x^{i}$ only. With this metric assumption, we consider a charged static dust source with the matter part of the energy-momentum tensor given by,

$$
T_{\mu \nu}^{M}=\rho u_{\mu} u_{\nu},
$$

where $u_{\mu}=-\frac{1}{\lambda} \delta_{\mu}^{0}$ since the dust is static. The fourpotential due to the static charged dust is in the form $A_{\mu}=$ $A_{0}\left(x^{i}\right) \delta_{\mu}^{0}$, so the current four-vector is given by,

$$
J^{\mu}=\sigma\left(x^{i}\right) u^{\mu}=\sigma\left(x^{i}\right) \lambda \delta_{\mu}^{0},
$$

where $\sigma\left(x^{i}\right)$ is the charge density of the dust. Therefore the electromagnetic and the Maxwell energy-momentum tensors are given by,

$$
\begin{gathered}
F_{\mu \nu}=\nabla_{\nu} A_{\mu}-\nabla_{\mu} A_{\nu}, \\
M_{\mu \nu}=\frac{1}{4 \pi}\left(F_{\mu \alpha} F_{\nu}^{\alpha}-\frac{1}{4} F_{\alpha \beta} F^{\alpha \beta} g_{\mu \nu}\right) .
\end{gathered}
$$


Using the Einstein tensor calculated from the metric (2) and the field equations $G_{\mu \nu}=8 \pi\left(T_{\mu \nu}^{M}+M_{\mu \nu}\right)$ with (3)(6) we get,

$$
A_{0}=\frac{\kappa}{\lambda}, \quad \kappa= \pm 1 .
$$

Then plugging the above equation back into the Einstein equations give,

$$
\nabla^{2} \lambda+4 \pi \rho \lambda^{3}=0
$$

Therefore the Einstein-Maxwell equations reduce to a nonlinear type of Poisson's equation. To find the equation satisfied by the charge density $\sigma\left(x^{i}\right)$ we use the Maxwell equations $\nabla_{\nu} F^{\mu \nu}=4 \pi J^{\mu}$ with (4) and (5) to get,

$$
\nabla^{2} \lambda+4 \pi \kappa \sigma \lambda^{3}=0 .
$$

Comparing (8) and (9) gives $\rho=\kappa \sigma$ which shows that the dust source is extremely charged.

One can solve the reduced Einstein-Maxwell Eqs. (8) and (9) for the extremely charged dust restricted to a thin shell as given in [5]. Letting $S$ be a regular surface in $\mathbb{R}^{3}$ defined by $S=\left[(x, y, z) \in \mathbb{R}^{3} ; F(x, y, z)=0\right]$, the matter density can be written as,

$$
\rho(\mathbf{r})=\rho_{0}(\mathbf{r}) \delta(F(\mathbf{r})),
$$

where $\rho_{0}$ is the matter distribution on the shell $S$. Generalizing the method given in [5] we can set,

$$
\lambda(\mathbf{r})=\lambda_{0}(\mathbf{r})-\lambda_{1}(\mathbf{r}) \Theta(F)-\lambda_{2}(\mathbf{r})(1-\Theta(F)),
$$

where $\Theta(F)$ is the Heaviside step function. The above choice can be made since the spacetime $\mathcal{M}$ is now divided into two disjoint spacetimes $\mathcal{M}^{+}$and $\mathcal{M}^{-}$by $S$ where the metric functions $\lambda$ are $\lambda_{0}-\lambda_{1}$ and $\lambda_{0}-\lambda_{2}$ respectively. We also require that the following equations are satisfied,

$$
\begin{gathered}
\nabla^{2} \lambda_{0}=\nabla^{2} \lambda_{1}=\nabla^{2} \lambda_{2}=0, \\
\left.\left(\lambda_{1}-\lambda_{2}\right)\right|_{S}=0 .
\end{gathered}
$$

These follow from the fact that in $\mathcal{M}^{+}$and $\mathcal{M}^{-}$the Laplace's equation is satisfied as can be seen from (8) (Since $\rho=0$ in $\mathcal{M}^{+}$and $\mathcal{M}^{-}$) and the metric must be continuous across the shell. Inserting (11) into (8) and using the conditions (12) and (13) we get,

$$
\rho_{0}=\left.\frac{\left(\nabla \lambda_{1}-\nabla \lambda_{2}\right) \cdot \nabla F}{4 \pi \lambda_{0}^{3}}\right|_{S} .
$$

Let $S$ be the sphere $F=r-a=0$. One can choose $\lambda_{2}=$ $0, \lambda_{1}=\lambda_{3}(\theta, \phi)\left(\frac{1}{a}-\frac{1}{r}\right)$ which clearly satisfies (12) and (13). Using (14) gives,

$$
\rho_{0}(\theta, \phi)=\frac{\lambda_{3}(\theta, \phi)}{4 \pi a^{2}\left(\left.\lambda_{0}\right|_{S}\right)^{3}},
$$

where $\lambda_{3}(\theta, \phi)$ satisfies,

$$
\left[\frac{1}{r^{2} \sin \theta} \frac{\partial}{\partial \theta}\left(\sin \theta \frac{\partial}{\partial \theta}\right)+\frac{1}{r^{2} \sin ^{2} \theta} \frac{\partial^{2}}{\partial \phi^{2}}\right] \lambda_{3}(\theta, \phi)=0 .
$$

At this point we would like to remark that the above Eq. (15) can also be obtained by using the Israel junction conditions [9]. However, when Israel junction conditions are used the surface energy-momentum tensor has the components,

$$
S_{t t}=\frac{\lambda_{3}(\theta, \phi)}{4 \pi a^{2}\left(\lambda_{0} I_{S}\right)^{4}}, \quad S_{\theta \theta}=S_{\phi \phi}=0 .
$$

So the matter density $\rho_{I}$ on $S$ is given by using the fact that $S_{t t}=\rho_{I} u_{t} u_{t}=\left.\frac{\rho_{I}}{\lambda_{0}^{2}}\right|_{S}$,

$$
\rho_{I}(\theta, \phi)=\frac{\lambda_{3}(\theta, \phi)}{4 \pi a^{2}\left(\left.\lambda_{0}\right|_{S}\right)^{2}} .
$$

As can be seen from (17) in Israel method we get $\lambda_{0}^{2}$ in the denominator instead of $\lambda_{0}^{3}$ as in (15) which is due to a dimensional scaling. The total mass on the shell can be written as an integral of $\rho_{0} \delta(F)$ over the 3 spatial dimensions which is equal to the integral of $\rho_{I}$ over the 2 dimensional surface, that is,

$$
\int_{\mathcal{V}_{3}} \rho_{0} \delta(F) \lambda^{3} d^{3} x=\int_{S_{2}} \rho_{I} \lambda^{2} d^{2} x
$$

So we get the same total mass on the shell in either method which means that there is no ambiguity.

When the interior of the sphere $S$ is chosen as a flat spacetime, we can set $\lambda_{0}=1$ and $\lambda_{3}=m_{0}$ as constant which turns out to be the mass of the shell. So (15) becomes,

$$
\rho_{0}=\frac{m_{0}}{4 \pi a^{2}} .
$$

So the interior and exterior metric functions are given by,

$$
\begin{gathered}
\lambda_{\text {out }}=1-\frac{m_{0}}{a}+\frac{m_{0}}{r}, \\
\lambda_{\text {in }}=1,
\end{gathered}
$$

where $m_{0}$ is the mass of the shell as can clearly be seen from (19). This solution represents the ERN solution exterior to a spherical shell with flat interior. Letting $r=$ $\left(R-m_{0}\right) / \beta$ where $\beta \equiv 1-m_{0} / a$ with $m_{0} \neq a$ assumed, the exterior metric can be written as,

$$
d s^{2}=-\beta^{2}\left(1-\frac{m_{0}}{R}\right)^{2} d t^{2}+\left(1-\frac{m_{0}}{R}\right)^{2} d R^{2}+R^{2} d \Omega^{2},
$$

which is the conventional form of ERN metric (after a scaling). As we noted earlier, we aim to remove the singularity of an ERN spacetime. Choosing $m_{0}<a$ we see from (20) that $\lambda_{\text {out }}>0$ is always satisfied. Comparing (22), the relation $r=\left(R-m_{0}\right) / \beta$ and (20) one can realize that the singularity of ERN spacetime is at $\lambda_{\text {out }}=0$ (which corre- 
sponds to $R=0$ ) and the horizon is at $r=0$ (which corresponds to $R=m_{0}$ ). This means that by restricting the matter on the shell with $m_{0}<a$ we remove the both the singularity and the horizon of the ERN spacetime. In the case $m_{0}>a(20), \lambda_{\text {out }}=0$ is not excluded. The case $m_{0}=a$ represents the Levi-Civita-Bertotti-Robinson (LCBR) spacetime outside the shell and flat spacetime inside. By letting $r=m_{0}^{2} / R$ the exterior metric is obtained as,

$$
d s^{2}=\frac{m_{0}}{R^{2}}\left[-d t^{2}+d R^{2}+R^{2} d \Omega^{2}\right],
$$

as the usual conformally flat LCBR metric. At this point we would like to note that in our analysis the limiting case $a \rightarrow$ 0 does not exist since in that limit the matter density (10) would become $\rho(\mathbf{r})=\rho_{0}(\mathbf{r}) \delta(\mathbf{r})$ and this choice is inconsistent with the Einstein-Maxwell Eqs. (8) and (9). If we had the Newtonian theory, such a limit mathematically would be consistent with the Poisson's equation. The nonexistence of the $a \rightarrow 0$ limit is in agreement with the results of [10].

To remove the singularities of a MP spacetime possessing $N$ ERN black holes discussed in [3], we place the matter source on thin shells. A MP spacetime possessing $N$ ERN black holes has the metric function $\lambda$ given by,

$$
\lambda=1+\sum_{j=1}^{N} \frac{m_{i}}{\left|\mathbf{r}-\mathbf{a}_{\mathbf{i}}\right|},
$$

where $\mathbf{a}_{\mathbf{i}}$ is the position of the $i^{\text {th }}$ ERN black hole. Such a spacetime contains $N$ number of singularities. We can place the extremely charged dust on $N$ spatially separated shells that do not intersect, instead of considering point sources. Generalizing the choice we made in (10) we can write the matter density of the spacetime as,

$$
\rho(\mathbf{r})=\sum_{j=1}^{N} \rho_{0 j}(\mathbf{r}) \delta\left(F_{j}\right)
$$

where $\rho_{0 j}$ is the matter distribution on the $j^{\text {th }}$ shell defined by $S_{j}: F_{j}(\mathbf{r})=0$. We can also generalize the choice for $\lambda$ as,

$$
\lambda=\lambda_{0}-\lambda_{e} \prod_{j=1}^{N} \Theta\left(F_{j}\right)-\sum_{j=1}^{N} \lambda_{j}\left[1-\Theta\left(F_{j}\right)\right],
$$

so that the metric function inside the $j^{\text {th }}$ shell is given by,

$$
\lambda_{j}^{\text {in }}=\lambda_{0}-\lambda_{j} \quad \forall j=1, \ldots, N .
$$

Moreover the metric function exterior to all of the $N$ shells is given by,

$$
\lambda^{\mathrm{ext}}=\lambda_{0}-\lambda_{e} .
$$

The functions $\lambda_{0}, \lambda_{j}$ and $\lambda_{e}$ satisfy the following,

$$
\nabla^{2} \lambda_{0}=\nabla^{2} \lambda_{j}=\nabla^{2} \lambda_{e}=0,
$$

$$
\left.\left(\lambda_{e}-\lambda_{j}\right)\right|_{S_{j}}=0 \quad \forall j=1, \ldots, N,
$$

where we again use the fact that the Laplace's equation is satisfied in source free regions and the metric across the shells must be continuous. Using (26)-(30) we calculate $\nabla^{2} \lambda$ as,

$$
\nabla^{2} \lambda=\sum_{j=1}^{N}\left(\nabla \lambda_{j}-\nabla \lambda_{e}\right) \cdot \nabla F_{j} \delta\left(F_{j}\right) .
$$

Using the fact that $\delta\left(F_{j}\right) \Theta\left(F_{k}\right)=\delta\left(F_{j}\right)$ for $k \neq j, \rho \lambda^{3}$ is given by,

$$
\rho \lambda^{3}=\sum_{j=1}^{N} \rho_{0 j}\left(\lambda_{0}-\lambda_{j}\right)^{3} \delta\left(F_{j}\right) .
$$

Then inserting (31) and (32) into (8) we get,

$$
\rho_{0 j}=\left.\frac{\left(\nabla \lambda_{e}-\nabla \lambda_{j}\right) \cdot \nabla F_{j}}{4 \pi\left(\lambda_{0}-\lambda_{j}\right)^{3}}\right|_{S_{j}} .
$$

As an illustration we consider the case of two spherical shells with radii $r_{1}$ and $r_{2}$ and the centers located at $\mathbf{a}_{1}$ and $\mathbf{a}_{2}$. Our aim is to obtain the exterior MP solution with the metric function given in (24) so that the singularities can be removed. The equations defining the spherical shells are given by $F_{1}=\left|\mathbf{r}-\mathbf{a}_{1}\right|-r_{1}=0$ and $F_{2}=\left|\mathbf{r}-\mathbf{a}_{2}\right|-$ $r_{2}=0$. Making analogy with (24) and the single shell solution (20) we choose,

$$
\lambda_{e}=\frac{m_{1}}{r_{1}}+\frac{m_{2}}{r_{2}}-\frac{m_{1}}{\left|\mathbf{r}-\mathbf{a}_{\mathbf{1}}\right|}-\frac{m_{2}}{\left|\mathbf{r}-\mathbf{a}_{\mathbf{2}}\right|} .
$$

Appropriate choices for $\lambda_{1}$ and $\lambda_{2}$ can be made by the following forms using (29) and (30),

$$
\begin{aligned}
& \lambda_{1}=\frac{m_{2}}{r_{2}}-\frac{m_{2}}{\left|\mathbf{r}-\mathbf{a}_{2}\right|}, \\
& \lambda_{2}=\frac{m_{1}}{r_{1}}-\frac{m_{1}}{\left|\mathbf{r}-\mathbf{a}_{\mathbf{1}}\right|},
\end{aligned}
$$

which in turn gives the full metric functions with the choice $\lambda_{0}=1$ as,

$$
\begin{gathered}
\lambda^{\mathrm{ext}=1-} \frac{m_{1}}{r_{1}}-\frac{m_{2}}{r_{2}}+\frac{m_{1}}{\left|\mathbf{r}-\mathbf{a}_{1}\right|}+\frac{m_{2}}{\left|\mathbf{r}-\mathbf{a}_{2}\right|}, \\
\lambda_{1}^{\text {in }}=1-\frac{m_{2}}{r_{2}}+\frac{m_{2}}{\left|\mathbf{r}-\mathbf{a}_{2}\right|}, \\
\lambda_{2}^{\text {in }}=1-\frac{m_{1}}{r_{1}}+\frac{m_{1}}{\left|\mathbf{r}-\mathbf{a}_{1}\right|} .
\end{gathered}
$$

The above choices describe an exterior MP spacetime and ERN spacetimes inside each shell. Let $\mathbf{a}_{\mathbf{1}}=-a \mathbf{e}_{\mathbf{z}}$ and $\mathbf{a}_{2}=a \mathbf{e}_{\mathbf{z}}$ so that the centers of the shells are on the $\mathrm{z}$ axis with $r_{1}+r_{2}<2 a$. The surface matter distributions are obtained from (33) with $N=2$ as, 


$$
\begin{gathered}
\rho_{01}=\frac{m_{1}}{4 \pi r_{1}^{2}}\left[1-\frac{m_{2}}{r_{2}}+\frac{m_{2}}{\sqrt{r_{1}^{2}-4 a r_{1} \cos \theta_{1}+4 a^{2}}}\right]^{-3}, \\
\rho_{02}=\frac{m_{2}}{4 \pi r_{2}^{2}}\left[1-\frac{m_{1}}{r_{1}}+\frac{m_{1}}{\sqrt{r_{2}^{2}+4 a r_{2} \cos \theta_{2}+4 a^{2}}}\right]^{-3},
\end{gathered}
$$

where $\theta_{1}$ and $\theta_{2}$ are spherical coordinates on $S_{1}$ and $S_{2}$ with their centers are taken as if origin. It can clearly be seen from (40) and (41) that the existence of a second shell disturbs the uniform matter distribution on the other shell so that the system stays at equilibrium. The above equations can be obtained by Israel method as we discussed before, where one obtains the second power of the term in the denominator instead of the third power as in (40) and (41) which is due to dimensional scaling. One can obtain (24) by letting $\beta \equiv 1-\frac{m_{1}}{r_{1}}-\frac{m_{2}}{r_{2}}$ in (37) so $\lambda^{\text {ext }}$ becomes,

$$
\lambda^{\mathrm{ext}}=\beta\left(1+\frac{\bar{m}_{1}}{\left|\mathbf{r}-\mathbf{a}_{\mathbf{1}}\right|}+\frac{\bar{m}_{2}}{\left|\mathbf{r}-\mathbf{a}_{\mathbf{2}}\right|}\right)=\beta \bar{\lambda}_{\mathrm{ext}}
$$

where $\bar{m}_{1}=m_{1} / \beta$ and $\bar{m}_{2}=m_{2} / \beta$. The MP spacetime possessing two ERN black holes has two horizons each described as a surface enclosing the points where the black holes are located [3]. These singularities are defined by the vanishing of $\lambda^{\text {ext }}$. So choosing the masses and the radii of the shells such that $m_{1} / r_{1}+m_{2} / r_{2}<1$, we guarantee that $\lambda^{\text {ext }}>0$ from (37), which means that by this choice we remove the singularities. Note that $\lambda_{1}^{\text {in }}$ in (38) and $\lambda_{2}^{\text {in }}$ in (39) can never vanish in their domains of definition. The case $m_{1} / r_{1}+m_{2} / r_{2}>1$ is irrelevant since we can not remove the singularities of the MP spacetime. The case $m_{1} / r_{1}+m_{2} / r_{2}=1$ should correspond to two mass generalization of the LCBR metric. Thus we obtained the double ERN black-hole solutions with singularities removed. (Note that we obtained the solution with a rescaling factor $\beta$ ). At this point we remark that MP-solutions with multiple spherical dust shells as sources, can not have flat interiors as we showed in (38) and (39). Moreover, the shells disturb each other and cause angular dependence of matter density on the shells. One can see from (40) and (41) that the second shell $\left(S_{2}\right)$ has maximum matter density at its north pole and the first shell $\left(S_{1}\right)$ has its maximum matter density at its south pole. Such a configuration puts the system in equilibrium. One can also calculate the masses of the shells by integrating (40) and (41) on $S_{1}$ and $S_{2}$ respectively as in (18) which gives the mass of the first shell as $m_{1}$ and the second shell as $m_{2}$ which is expected. We again note that the limits $r_{1} \rightarrow 0$ and $r_{2} \rightarrow$ 0 do not exist since the matter density (25) is not consistent with the Einstein-Maxwell Eqs. (8) and (9). This means that we can not have a source density of the form,

$$
\rho(\mathbf{r})=m_{1} \delta\left(\mathbf{r}-\mathbf{a}_{1}\right)+m_{2} \delta\left(\mathbf{r}-\mathbf{a}_{2}\right) .
$$

This is again in total agreement with [10].

One can also consider other solutions to the Laplace's equation for the metric function $\lambda$ to obtain various spacetimes. It was shown that such spacetimes possess naked singularities [3]. One can remove these naked singularities by placing the extremely charged source on thin shells as we did in this work for multiple ERN black-hole solutions. The same procedure can also be applied to the stationary generalization of MP-spacetimes given by Israel-Wilson [11] and Perjés [12] to remove the naked singularities of these spacetimes.

\section{SHELL MODELS FOR THE KASTOR-TRASCHEN SPACETIMES}

It was shown that time-dependent generalizations of MP spacetimes exist and solutions corresponding to $N$ extremely charged comoving black holes in a de-Sitter background were considered in [6-8]. The idea of removing the singularities of $N$ comoving black-hole solutions by thin shells were considered briefly in [7] for testing the cosmic censorship conjecture. At this point we would like to extend the discussion given in [7] by the methods we discussed above.

The cosmological MP solution which is a timedependent generalization of the MP solution with metric (2) in cosmological coordinates is given by,

$$
d s^{2}=-\tilde{U}^{-2} d t^{2}+R(t)^{2} \tilde{U}^{2} h_{i j} d x^{i} d x^{j},
$$

where $R(t)$ is the scale factor and $h_{i j}$ is an Euclidean metric. For a single extreme Reissner-Nördstrom-deSitter (ERNdS) black hole with its charge equal to its mass $(Q=M)$ the metric function $\tilde{U}$ in the metric (43) is given by,

$$
\tilde{U}=1+e^{-H t} \frac{M}{r} .
$$

We can obtain the ERNdS solution in static coordinates by following the transformation given in [6] Given the metric (43) with $\tilde{U}$ being arbitrary we consider a comoving charged dust as source. As before, we assume the fourpotential of the form $A_{\mu}=A_{0} \delta_{\mu}^{0}$ and $u_{\mu}=-\frac{1}{\tilde{U}} \delta_{\mu}^{0}$ since the dust is comoving (i.e. static in cosmological coordinates). Then the Einstein equations with positive cosmological constant become,

$$
G_{\mu \nu}=8 \pi\left(\rho u_{\mu} u_{\nu}+M_{\mu \nu}\right)+\Lambda g_{\mu \nu},
$$

where $M_{\mu \nu}$ is the Maxwell tensor given as in (6). Then the Einstein equations (45) give,

$$
\begin{gathered}
\left(\frac{\dot{R}}{R}\right)^{2}=\frac{\Lambda}{3}, \\
\nabla^{2} \tilde{U}+4 \pi \rho R(t)^{2} \tilde{U}^{3}=0,
\end{gathered}
$$




$$
\tilde{U}=1+\frac{1}{R(t)} \lambda(x)
$$

where dot over $R$ represents derivative with respect to $t$ and $\lambda(x)$ is independent of $t$. The above equations are obtained by separating the cosmological and electromagneticmatter parts of the Einstein equations. One can note that (46) is just the Friedmann equation for a flat cosmology. Solving (46) yields $R(t)=e^{H t}$ where $H= \pm \sqrt{3 / \Lambda}$. As discussed in [6-8] negative $H$ corresponds to black-hole spacetimes while positive $H$ corresponds to white hole spacetimes. We consider negative $H$ for the rest of the paper $(H=-|H|)$. The Maxwell equations $\nabla_{\nu} F^{\mu \nu}=$ $4 \pi J^{\mu}$ give,

$$
\nabla^{2} \tilde{U}+\frac{4 \pi \sigma}{\kappa} R(t)^{2} \tilde{U}^{3}=0
$$

with $\kappa= \pm 1$ and $\sigma$ being the charge density of the comoving dust. Comparing (47) and (49) we get $\rho=\kappa \sigma$ as before, so we conclude that the dust is extremely charged. Letting $d \tau=R(t) d t$ we can write the metric (43) as,

$$
d s^{2}=-U^{-2} d \tau^{2}+U^{2} h_{i j} d x^{i} d x^{j} .
$$

where

$$
\begin{gathered}
U=H \tau+\lambda(x), \\
\nabla^{2} U+4 \pi \rho U^{3}=0 .
\end{gathered}
$$

One can see from the Einstein Eqs. (52) that the product $\rho U^{3}$ must be time independent. This is clear from the fact $\nabla^{2} U$ is time independent as can be seen from (51) and to have the Eq. (52) satisfied for all times we must have $\rho U^{3}$ to be time independent.

The above derivation of Einstein-Maxwell equations for a charged dust was given in [7]. Our treatment of the shell model will be parallel to which we considered in the previous part of this work, that is different from the shells considered in [7]. We start with a dust model and then take its limit to thin shells with the use of Dirac delta functions for the matter density. Since (47) and (49) are totally same with Eqs. (8) and (9) we can consider similar multishell models with the matter density given in (25) and the metric function $U$ as in (26) (Clearly we just replace $\lambda$ with $U$ ). One difference is that the metric function $U$ is time dependent via $H \tau$. However, as can be seen from (51) and (52) this time dependence does not affect Einstein-Maxwell equations. In source free regions we again have the Laplace's equation satisfied. The equation of each surface defining the shells are again given by $F_{j}(\mathbf{r})=0$. Thus we follow the lines (25) to (33) to get the same dependence for the surface matter density.
As an example, we again consider two spherical shells with radii $r_{1}, r_{2}$ and with centers located at $\mathbf{a}_{1}, \mathbf{a}_{2}$. The equations describing the shells are given by,

$$
F_{1}=\left|\mathbf{r}-\mathbf{a}_{1}\right|-r_{1}=0, \quad F_{2}=\left|\mathbf{r}-\mathbf{a}_{2}\right|-r_{2}=0 .
$$

Because of linearity of Laplace's equation, we extend the single ERNdS solution in cosmological coordinates given in (50) to the double ERNdS case for the exterior metric function as,

$$
U^{\mathrm{ext}}=H \tau-\frac{m_{1}}{r_{1}}-\frac{m_{2}}{r_{2}}+\frac{m_{1}}{\left|\mathbf{r}-\mathbf{a}_{\mathbf{1}}\right|}+\frac{m_{2}}{\left|\mathbf{r}-\mathbf{a}_{\mathbf{2}}\right|},
$$

where we have chosen $U_{0}=H \tau$ in (28). Then (38) and (39) suggests,

$$
\begin{aligned}
& U_{1}^{\text {in }}=H \tau-\frac{m_{2}}{r_{2}}+\frac{m_{2}}{\left|\mathbf{r}-\mathbf{a}_{2}\right|}, \\
& U_{2}^{\text {in }}=H \tau-\frac{m_{1}}{r_{1}}+\frac{m_{1}}{\left|\mathbf{r}-\mathbf{a}_{1}\right|},
\end{aligned}
$$

for the metrics inside the first and the second shells. Clearly the choices (54)-(56) satisfy the required conditions (29) and (30) for $N=2$. We have mentioned above that $\rho U^{3}$ must be time independent which is satisfied in our multishell model since this product is given with the use of (25), (26), (55), and (56) as,

$$
\rho U^{3}=\rho_{01}\left(U_{1}^{\mathrm{in}}\right)^{3} \delta\left(F_{1}\right)+\rho_{02}\left(U_{2}^{\mathrm{in}}\right)^{3} \delta\left(F_{2}\right) .
$$

Since $F_{1,2}$ are time independent and $\rho_{01,2} U_{1,2}^{\text {in }}$ will be shown to be time independent below.

Again choosing $\mathbf{a}_{\mathbf{1}}=-a \mathbf{e}_{\mathbf{z}}$ and $\mathbf{a}_{\mathbf{2}}=a \mathbf{e}_{\mathbf{z}}$ such that $r_{1}+r_{2}<2 a$ the surface matter distributions are calculated from (33) as,

$$
\begin{aligned}
& \rho_{01}=\frac{m_{1}}{4 \pi r_{1}^{2}}\left[H \tau-\frac{m_{2}}{r_{2}}+\frac{m_{2}}{\sqrt{r_{1}^{2}-4 a r_{1} \cos \theta_{1}+4 a^{2}}}\right]^{-3}, \\
& \rho_{02}=\frac{m_{2}}{4 \pi r_{2}^{2}}\left[H \tau-\frac{m_{1}}{r_{1}}+\frac{m_{1}}{\sqrt{r_{2}^{2}+4 a r_{2} \cos \theta_{2}+4 a^{2}}}\right]^{-3},
\end{aligned}
$$

where the definitions of $\theta_{1}$ and $\theta_{2}$ are given as before. As can be seen from above, $\rho_{01}$ and $\rho_{02}$ are time dependent but when they are integrated on the surface of each shell (the term $\rho_{01,2}\left(U_{1,2}^{\text {in }}\right)^{3}$. will appear in the integral which is time independent), they give constant masses for the shells as $m_{1}$ and $m_{2}$ which is in consistence with the result of Bonnor [8] . If we had a single shell, the interior of the shell would be de-Sitter. But for the case of double shells (and for $N>2$ also) as can be seen from (55) and (56) the interior of the shells are ERNdS. Unless this choice is made, the continuity condition of the metric across the shells will be violated. This fact was not realized in [7] where the interior of the shells were considered as de-Sitter. 
To remove the singularities of the cosmological multiblack hole solutions we again restrict the matter on thin shells. For that purpose, we assume that the thin shells are formed at $\tau=0$ with $H<0$ so from (54) one can see that $U^{\text {ext }}$ never vanishes for later times $\tau \geq 0$. This means that the exterior metric is singularity free for all times. Moreover the interior of the shells described by (55) and (56) can also never vanish for the choice we made. Thus the whole spacetime becomes regular. The spacetime distances $\|P Q\|_{g}$ between two neighboring (space) points $P$ and $Q$ is given by $\|P Q\|_{g}=|U|\|P-Q\|$ where $\|P-Q\|$ is the distance in $\mathbb{R}^{3}$, and $U$ is given in (51). Hence in exterior and interior spacetimes the space distances increase with constant (Hubble) speed. For this reason the shells inflate without collision, because the distances among them also increase.

There is also another interesting possibility of constructing regular spacetimes by considering $H>0$ for $\tau \leq 0$ and $H<0$ for $\tau \geq 0$. With these choices one can see that $U^{\text {ext }}$, $U_{1}^{\text {in }}$ and $U_{2}^{\text {in }}$ never vanishes and the spacetime metric is continuous at $\tau=0$. In this model, the Hubble constant $H$ changes sign in each universe separated by $\tau=0$ which causes a delta-function type of singularity at this hypersurface. Spacetimes (interior and exterior spacetime regions) are contracting for $\tau \leq 0$ and expanding for $\tau \geq 0$.

Our method can be applied also to higher dimensional generalizations of the Majumdar-Papapetrau-KastorTraschen solutions [13] to study brane world creations and collisions [14].

\section{CONCLUSION}

We considered thin mass shell models for the Majumdar-Papapetrou and Kastor-Traschen spacetimes. In both cases we found exact solutions of the field equations describing gravitational fields of the extremely charged $N$-number mass shells. Shell interiors are also curved spacetimes matching smoothly to the exteriors through (three dimensional) infinitely thin shells. We found the mass ( also the charge) densities on each shell. The solutions obtained this way are free of singularities. For the case of Kastor-Traschen spacetimes choosing the sign of the Hubble constant $H$ properly we showed that shells are moving away from each other with constant velocity.

Shell models in higher dimensional theories seem to be also very interesting. In order that our method to be applicable to such theories one has to modify Einstein field equations with the inclusion of a dust matter as we did in this work where the mass is distributed on thin shells. In this case the shells are also higher dimensional. In the case of five dimensions for instance the thin shells are four dimensional spacetimes. Our work on this matter will be communicated elsewhere.

\section{ACKNOWLEDGMENTS}

This work is partially supported by the Scientific and Technical Research Council of Turkey and by the Turkish Academy of Sciences.
[1] S. D. Majumdar, Phys. Rev. 72, 390 (1947).

[2] A. Papapetrou, Proc. Roy. Soc. Irish. Acad. A 51, 191 (1947).

[3] J. B. Hartle and S. W. Hawking, Commun. Math. Phys. 26, 87 (1972).

[4] M. Gürses, Phys. Rev. D 58, 044001 (1998).

[5] M. Gürses, in Proceedings of the international seminar: Current Topics in Mathematical Cosmology (World Scientfic, Singapore, 1998).

[6] D. Kastor and J. Traschen, Phys. Rev. D 47, 5370 (1993).

[7] D. R. Brill, G. T. Horowitz, D. Kastor, and J. Traschen, Phys. Rev. D49, 840 (1994).
[8] W. B. Bonnor, Class.l Quant. Grav. 17, 3935 (2000).

[9] W. Israel, Nuovo Cimento B 44, 1 (1966).

[10] R. Geroch and J. Traschen, Phys. Rev. D 36, 1017 (1987).

[11] W. Israel and G. A. Wilson, J. Math. Phys. (N.Y.) 13, 865 (1972).

[12] Z. Perjés, Phys. Rev. Lett., 27, 1668 (1971).

[13] T. Maki and K. Shiraishi, Class. Quant. Grav. 10, 2171 (1993).

[14] G. W. Gibbons, H. Lü, and C. N. Pope, Phys. Rev. Lett. 94, 131602 (2005). 